\title{
Normal Pregnancy Is Associated with Changes in Central Hemodynamics and Enhanced Recruitable, but Not Resting, Endothelial Function
}

\author{
Juan Torrado, ${ }^{1}$ Yanina Zócalo, ${ }^{1}$ Ignacio Farro, ${ }^{1}$ Federico Farro, ${ }^{1}$ Claudio Sosa, ${ }^{2}$ \\ Santiago Scasso, ${ }^{2}$ Justo Alonso, $^{2}$ and Daniel Bia ${ }^{1}$ \\ ${ }^{1}$ Centro Universitario de Investigación, Innovación y Diagnóstico Arterial (CUiiDARTE), Physiology Department, Faculty of Medicine, \\ Republic University, General Flores 2125, 11800 Montevideo, Uruguay \\ ${ }^{2}$ Department of Obstetrics and Gynecology "C", Pereira-Rossell Hospital, Faculty of Medicine, Republic University, Br. Artigas 1550, \\ 11600 Montevideo, Uruguay
}

Correspondence should be addressed to Daniel Bia; dbia@fmed.edu.uy

Received 23 June 2015; Accepted 18 August 2015

Academic Editor: Padma Murthi

Copyright (C) 2015 Juan Torrado et al. This is an open access article distributed under the Creative Commons Attribution License, which permits unrestricted use, distribution, and reproduction in any medium, provided the original work is properly cited.

Introduction. Flow-mediated dilation (FMD), low flow-mediated constriction (L-FMC), and reactive hyperemia-related changes in carotid-to-radial pulse wave velocity $(\triangle \mathrm{PWV} c r \%)$ could offer complementary information about both "recruitability" and "resting" endothelial function (EF). Carotid-to-femoral pulse wave velocity (PWVcf) and pulse wave analysis-derived parameters (i.e., AIx@75) are the gold standard methods for noninvasive evaluation of aortic stiffness and central hemodynamics. If healthy pregnancy is associated with both changes in resting and recruitable EF, as well as in several arterial parameters, it remains unknown and/or controversial. Objectives. To simultaneously and noninvasively assess in healthy pregnant (HP) and nonpregnant (NP) women central parameters in conjunction with "basal and recruitable" EF, employing new complementary approaches. Methods. HP ( $n=11,34.2 \pm 3.3$ weeks of gestation) and age- and cardiovascular risk factors-matched NP $(n=22)$ were included. Aortic blood pressure (BP), AIx@75, PWVcf, common carotid stiffness, and intima-media thickness, as well as FMD, L-FMC, and $\Delta$ PWVcr \%, were measured. Results. Aortic BP, stiffness, and AIx@75 were reduced in HP. $\triangle \mathrm{PWVcr \%}$ and FMD were enhanced in HP in comparison to NP. No differences were found in L-FMC between groups. Conclusion. HP is associated with reduced aortic stiffness, central BP, wave reflections, and enhanced recruitable, but not resting, EF.

\section{Introduction}

Arterial structure and function can now be simple and noninvasively assessed by different accurate methods, which have been extensively used in patients with cardiovascular risk factors. In this context, Celermajer et al's technique, commonly known as flow-mediated dilation (FMD), which utilizes the vasoreactivity test (VRT), has stood the test of time and remains the most popular method to assess endothelial function $[1,2]$. The VRT consists in positioning a pneumatic cuff around the forearm and provoking an arterial occlusion for five minutes (i.e., transient ischemia). This maneuver elicits an increase in blood flow in the brachial artery once the cuff is deflated (i.e., reactive hyperemia, $R H$ ), which subsequently stimulates endothelium to release several vasoactive biochemical factors (i.e., nitric oxide). Finally, locally produced factors result in a dilation of the brachial artery (measured by B-Mode ultrasound) [2] and a reduction in arterial stiffness (changes in pulse wave velocity [PWV] assessed by mechanotransducers [3]). The VRT has also been also applied in healthy pregnancy and in pregnancy-related diseases $[4,5]$. Independently of the setting, the magnitude of the arterial dilation is used as an indicator of endothelial function and healthy pregnant women show an enhanced vascular response evaluated by this method compared with healthy nonpregnant women [6, 7]. However, whereas FMD provides information about the "recruitability" of endothelial function (i.e., its responsiveness to a specific stimulus), it does 
not provide information concerning basal/tonic endothelial function (i.e., release of endothelial autacoids before FMD measures are initiated) [8]. In this context, Gori et al. described a novel index for assessing the response of the artery to low flow, which utilizes data obtained from the cuff occlusion period of an FMD scan [9]. Synonymous to FMD, the vasoconstriction observed under conditions of reduced blood flow has been named low-flow-mediated vasoconstriction (L-FMC) [9]. Inclusion of L-FMC data to traditional measurement of FMD could provide additional and/or complementary information, which, they propose, may improve the detection of patients with cardiovascular disease and profile the vascular response to exercise among healthy volunteers [10]. Whether healthy pregnancy is associated with changes in L-FMC remains to be established. In this context, it is also unknown if the integration of L-FMC into traditional FMD studies will be able to provide additional/complementary information among pregnant women.

In addition, changes in carotid-to-radial PWV (PWVcr) due to the same maneuver have been proposed as an alternative tool for the evaluation of recruitable endothelial function $[3,11]$. PWV is recognized as the "gold standard" parameter for the evaluation of regional arterial stiffness and has had a wide biomedical application $[12,13]$. A reduction in PWVcr values has been reported in response to VRT in healthy young adults [3] whereas a blunted reduction has been reported in pathophysiological circumstances, such as hypertension [14] and congestive heart failure [11]. A preliminary report suggested that changes in PWVcr due to VRT offer additional information of endothelial dynamics and thus could have a potential role in the assessment of endothelial function during pregnancy with a potential clinical application in predicting pregnancy-induced hypertension and preeclampsia [15].

Finally, there is still a lack of knowledge about the expected changes for several structural and functional parameters widely used in noninvasive arterial studies. In this regard, if pregnancy is associated with changes in central aortic pressure, wave reflections levels and/or elastic and muscular arteries stiffness remains to be determined. Changes in arterial structure and function of both maternal muscular and elastic arteries could be of value in accommodating pregnancy-induced increasing cardiac output and blood volume for a correct maternal-fetal hemodynamic interaction [16, 17]. Therefore, we here hypothesize that normal pregnancy, in contraposition to the nonpregnant status and particularly during the third trimester stage in which profound cardiovascular changes are expected, will evidence notable vascular adaptations (i.e., reduction in wave reflections levels and arterial stiffness), features capable of being assessed and quantified by using promising and complementary noninvasive arterial parameters.

In this context, the work aims were firstly to determine and analyze "basal and recruitable" endothelial function through the measurement of brachial artery FMD and LFMC and PWVcr RH-related changes in a group of healthy nonpregnant and pregnant women and, secondly, to determine noninvasively central and peripheral arterial parameters, by using validated and gold standard techniques.

\section{Methods}

2.1. Subjects, Demographic Characteristics, and Laboratory Samples. This was an analytic observational case-control study involving 11 healthy pregnant (HP) and 22 healthy nonpregnant women (NP). The HP women were recruited from the routine antenatal clinic where they were asked and agreed to participate in the study (convenience sampling). NP group was obtained from our database (CUiiDARTE Project and Centre, Republic University) once HP women were matched based on age, height, and cardiovascular risk factors [18-20]. Baseline demographic and anthropometric data were obtained by an obstetrician/physician during a clinical interview and exam, and laboratory samples were extracted prior to the examination. They were all healthy (with the exception of dyslipidemia in some of them) and without family history of premature cardiovascular disease. All HP women had uncomplicated pregnancies before and during the study. None of them received any vasoactive drugs.

Exclusion criteria for HP and NP included previous history of pregnancy-induced hypertension (including preeclampsia), gestational diabetes, or current chronic hypertension and/or diabetes mellitus. Significant unexplained proteinuria ( $>300 \mathrm{mg}$ total protein in a $24 \mathrm{~h}$ urine collection) developing in obstetric controls was also an exclusion criterion for HP. The definitions for pathologies used for exclusion criteria took into account the one recommended by the National Institute for Health and Clinical Excellence guidelines [21, 22].

Participants were asked to abstain from physical activity, tobacco products, and vitamin supplementation for at least 4 hours prior to the examination. The study protocol was approved by the Ethics Research Committee of the Republic University (Uruguay) and all participants gave written informed consent.

2.2. Baseline Noninvasive Arterial Evaluation. Subjects were instructed to lie in a left lateral position (particularly for $\mathrm{HP}$, to avoid vena cava compression by the uterus) in a temperature-controlled $\left(21^{\circ}-23^{\circ} \mathrm{C}\right)$ room, for at least $15 \mathrm{~min}$ utes, in order to establish stable hemodynamic conditions. Heart rate (HR) and right brachial (peripheral) systolic and diastolic blood pressure (pSBP and pDBP, resp.) were measured using an oscillometric device (Omron HEM-433INT Oscillometric System; Omron Healthcare Inc., Illinois, USA) at 5-8-minute intervals during the whole procedure. Mean blood pressure (MBP) was determined using classic empirical formula currently used at the peripheral level as pDBP plus one-third times of peripheral pulse pressure ( $\mathrm{pPP}=\mathrm{pSBP}-$ pDBP).

2.2.1. Carotid-to-Femoral Pulse Wave Velocity and Pulse Wave Analysis. The carotid-femoral pulse-wave velocity (PWVcf) was measured to analyze aortic regional stiffness. To this end, carotid and femoral artery waveforms were consecutively obtained with a high-fidelity applanation tonometer from the carotid and femoral regions simultaneously with continuous ECG monitoring (SphygmoCor 7.01, AtCor Medical, Sydney, Australia) (Figure 1). Then, carotid-femoral propagation time 


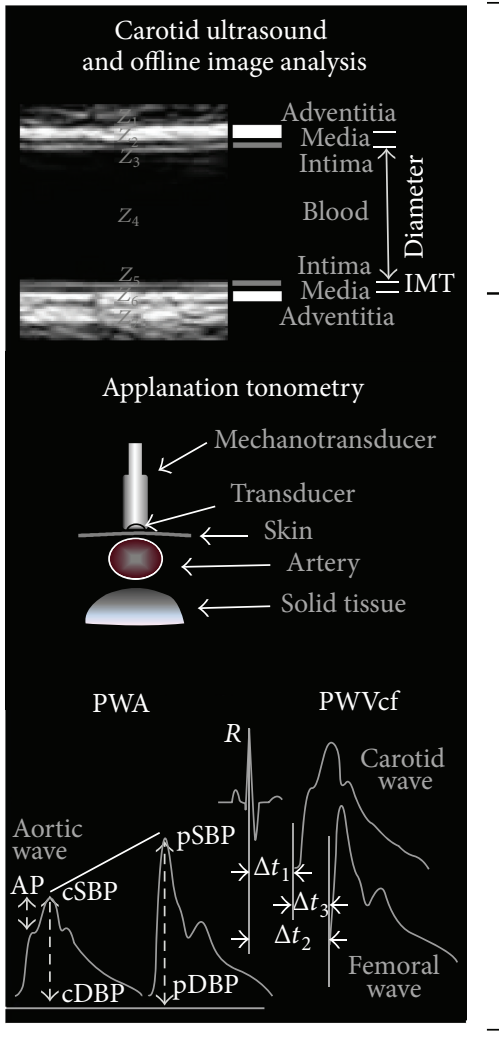

(a)

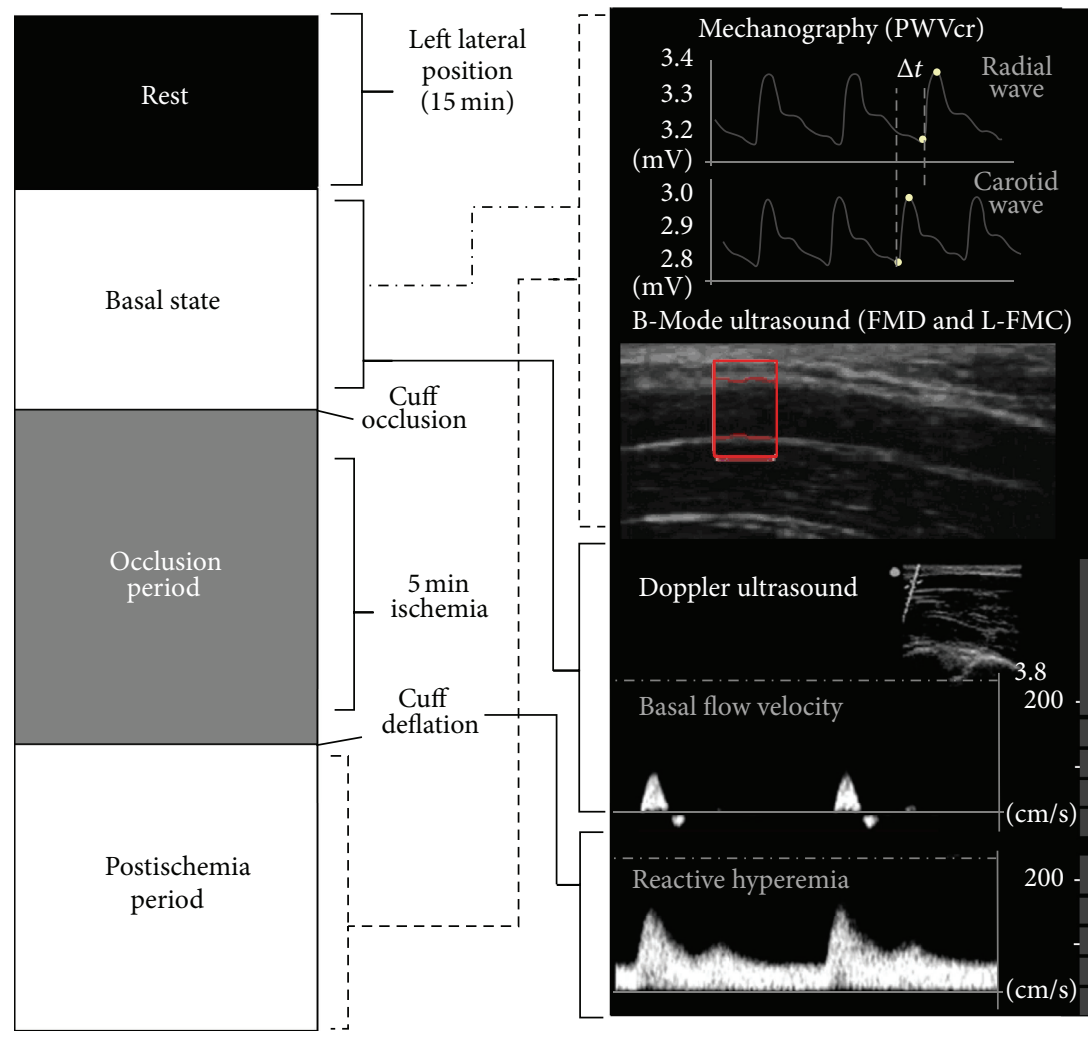

(b)

FIGURE 1: (a) Schema of the instrumental approach employed to acquire noninvasively arterial parameters in basal conditions. Employed techniques: carotid-to-femoral PWV and PWA (applanation tonometry) and carotid arterial diameter and CIMT (B-Mode ultrasound). (b) Representative diagram of the study protocol of Vasoreactivity Test (VRT) applied to evaluate changes in carotid-to-radial PWV and brachial arterial diameter. $Z$ : acoustic impedance; CIMT: carotid intima-media thickness; PWA: pulse wave analysis; PWVcf: carotid-tofemoral pulse wave velocity; $\Delta t_{1}, \Delta t_{2}$ : time delay between $R$ wave from ECG and central (carotid) foot wave and peripheral (femoral or radial) foot wave, respectively; $\Delta t_{3}$ : time delay between carotid foot wave and radial foot wave; AP: augmentation pressure; cSBP and pSBP: central and peripheral systolic blood pressure, respectively; cDBP and pDBP: central and peripheral diastolic blood pressure, respectively; PWVcr: carotid-to-radial pulse wave velocity; FMD: flow-mediated dilation; L-FMC: low flow-mediated constriction.

$\left(\Delta t_{3}\right)$ was determined by subtracting the time delay between the peak of $R$ wave of the ECG recording to femoral foot of the pressure waveform $\left(\Delta t_{2}\right)$ of the corresponding cardiac cycle and the time delay between the peak of $R$ wave to carotid foot of the pressure waveform $\left(\Delta t_{1}\right)$. The algorithm utilized to detect the so-called "foot of the wave" was the intersecting tangents, explained elsewhere [23]. Straight distance between the recording sites (carotid-to-femoral distance [C-F $\Delta x]$ ) was then carefully measured using tape on the body surface to reduce the influence of altered body contour in pregnancy. Finally, PWVcf was automatically calculated as the quotient between C-F $\Delta x$ and $\Delta t_{3}$ (Figure 1). The reported value of PWVcf for a subject was always the average of at least eight consecutive beats.

Pulse wave analysis (PWA) was used to assess central hemodynamics as well as systemic arterial stiffness and wave reflections. For this porpoise, mean radial artery waveform was obtained (through the acquisition of many cycles) with the applanation tonometer from the wrist, and a corresponding mean ascending aortic pressure waveform was generated with a validated generalized transfer function using the same mentioned customized software (SphygmoCor 7.01, AtCor Medical, Sydney, Australia) [24]. The radial pulse waveform was then calibrated using the diastolic and mean arterial pressure obtained at the brachial artery [12]. Central systolic, diastolic, and pulse pressure (cSBP, $\mathrm{cDBP}$, and $\mathrm{cPP}$, resp.), heart rate corrected central augmentation index (AIx@75, adjusted to a rate of 75 beats/minute), and amplification ratio (pPP/cPP) were determined with the integrated software.

\subsubsection{Carotid Artery Studies. Ultrasound assessment of} carotid arteries was based on the techniques and recommendations described in international consensus [25]. Highresolution B-Mode ultrasound images of both (right and left) common carotid arteries (CCA) were obtained using a lineararray transducer connected to a portable Ultrasound System (Probe L38e, 5-10 MHz, SonoSite, MicroMaxx, SonoSite Inc., 21919 30th Drive SE, Bothell, WA 98021, USA). Measurements (still images and video clips/cine loops) were digitally stored for offline analysis (Figure 1). Near and far walls were analyzed and images were obtained from anterior, lateral, and posterior angles. At first, a carotid plaque screening was 
performed, for which the definition used was a focal wall thickening at least $50 \%$ greater than that of the surrounding vessel, a thickening that protrudes into the lumen $0.5 \mathrm{~mm}$ or as a region with carotid intima-media thickness (CIMT) greater than $1.5 \mathrm{~mm}$ [25]. Then, longitudinal views of the CCAs were acquired and a video (cine loop) of at least 30 seconds was recorded and stored. The CIMT and beat-to-beat diameter waveforms were obtained and analyzed offline using a step-by-step border detection algorithm (based in changes in acoustic impedance $[Z]$ ), applied to each digitized image (Hemodyn-4M software, Buenos Aires, Argentina). A region $1.0 \mathrm{~cm}$ proximal to the carotid bulb was identified, and the far wall CIMT was determined as the distance between the lumen-intima and the media-adventitia interfaces (Figure 1). The software performs multiple automated or semiautomated measurements along the centimeter and averages them, increasing the accuracy of the measures.

The instantaneous mean diameter (from the leading edge of the near wall intima-media interface to the intima-media interface of the far wall) waveform was obtained during pulsation in order to obtain diastolic and systolic diameter. Then, the pressure strain or Peterson's elastic modulus $\left(E_{P}\right)$ was calculated relating these measures with central blood pressure as follows [12]:

$$
E_{P}=\frac{(\mathrm{cSBP}-\mathrm{cDBP})}{(\mathrm{SD}-\mathrm{DD}) / \mathrm{DD}}
$$

where cSBP, cDBP, SD, and DD are central systolic and diastolic blood pressure and carotid systolic and diastolic diameter, respectively (Figure 1 ). $E_{P}$ measures the ability of the arteries to change its dimensions in response to the pulse pressure caused by cardiac pulsatile ejection (pressure change required for (theoretic) $100 \%$ increase in diameter) [12, 26, 27].

\subsection{Vascular Reactivity: Resting and Recruitable Endothelial} Function. Once baseline noninvasive arterial evaluation was carried out, we utilized the theoretical basis, general protocol, and methodological aspects of the VRT recommended by the guidelines for the ultrasound assessment of endothelialdependent flow-mediated vasodilation of the brachial artery $[2,28]$. For this purpose, participants were submitted to five minutes of ischemia by occluding left radial and cubital arteries using a pneumatic cuff placed around the left forearm (just below the elbow to at least $50 \mathrm{~mm} \mathrm{Hg}$ above pSBP) and several parameters of vascular reactivity were measured before, during, and after ischemia (Figure 1). The parameters used for the evaluation of endothelial function are listed below.

2.3.1. L-FMC, FMD, and Shear Rate. Taking into account "gold standard" accepted methodology for the evaluation of endothelial function "recruitability" and simultaneously PWVcr measurement (see later), left brachial artery was visualized longitudinally above the antecubital crease using the same high resolution B-Mode ultrasound device mentioned earlier (Sonosite; MicroMaxx; USA) (Figure 1). Similarly, video sequences were recorded at rest (1 minute), during forearm occlusion ( 5 minutes), and after cuff deflation
(4 minutes). Subsequently and similarly to the processing of carotid images, recordings were analyzed offline using same automated step-by-step algorithm applied to each digitalized image that allows for the brachial diameter waveform obtainment and L-FMC and FMD calculation [29].

L-FMC was quantified as the percentage of change in brachial artery diastolic diameter (DD), considering the basal levels and the DD before cuff deflation:

$$
\mathrm{L}-\mathrm{FMC} \%=\frac{\mathrm{DD}_{\text {before cuff deflation }}-\mathrm{DD}_{\text {baseline }}}{\mathrm{DD}_{\text {baseline }}} \times 100
$$

FMD was quantified as the percentage of change in brachial DD, considering the basal levels and the maximal diastolic diameter after cuff deflation:

$$
\mathrm{FMD} \%=\frac{\mathrm{DD}_{\text {after cuff deflation }}-\mathrm{DD}_{\text {baseline }}}{\mathrm{DD}_{\text {baseline }}} \times 100
$$

In addition, Doppler signals were performed to acquire blood flow velocity in baseline conditions and at specific moments during the RH period. Doppler signals were used to obtain the brachial shear rate (SR) and its percentage of change, relating mean blood flow velocity $\left(V_{m}[\mathrm{~cm} / \mathrm{s}]\right)$ to brachial mean diameter $\left(D_{m}\right)$ according to the following equations:

$$
\begin{aligned}
\mathrm{SR} & =\frac{V_{m}}{D_{m}}, \\
\mathrm{SR} \% & =\frac{\mathrm{SR}_{\text {after cuff deflation }}-\mathrm{SR}_{\text {baseline }}}{\mathrm{SR}_{\text {baseline }}} \times 100 .
\end{aligned}
$$

SR is an estimate of shear stress without accounting for blood viscosity [30] and was obtained for the characterization of the endothelial stimulus. All measurements were done by the same trained operator. The study protocol is represented in Figure 1.

2.3.2. Carotid-to-Radial Pulse Wave Velocity. Noninvasively, carotid and radial pressure waveforms were simultaneously obtained using strain gauge mechanotransducers (Motorola MPX 2050, Motorola Inc., Corporate 1303 E. Algonquin Road, Schaumburg, Illinois 60196, USA) by placing them on the skin over the carotid and radial sites (left hemibody). PWVcr was determined taking into account the given distance between these arterial sites (C-R $\Delta x)$ and the time delay $(\Delta t)$ between the carotid and radial waveforms onset (Figure 1). The algorithm used for the detection of the foot waves was described and explained in previous work [31]. Although a four-minute recording postcuff release was obtained, one-minute postischemia was the specific moment where the analysis was especially taken, according to previous reports $[14,31,32]$. The PWVcr accepted variation coefficient was less than $7 \%$. 
PWVcr levels corresponding to baseline and to postischemia period were determined by averaging eight consecutive beats. After that, percent of change of PWVcr (with respect to basal levels) was quantified as follows:

$$
\Delta \mathrm{PWVcr} \%=\frac{\mathrm{PWVcr}_{\text {after cuff deflation }}-\mathrm{PWVcr}_{\text {baseline }}}{\mathrm{PWVcr} r_{\text {baseline }}}
$$$$
\times 100 \text {. }
$$

2.4. Statistics. The statistical analyses were performed using the Statistical Package for Social Sciences (version 19.0). All data were expressed as mean value $(\mathrm{MV}) \pm$ standard deviation (SD) and a $P<0.05$ indicates significant statistical differences. Comparisons between pregnant and nonpregnant women were performed using two-tailed unpaired Student $t$-test. Differences in prevalence were analyzed using $\chi^{2}$ Test with or without Yates's correction if appropriate. Differences in percentage of change of variables determined before and after the VRT (arterial diameter, PWV and shear rate) were evaluated using two-tailed paired Student $t$-test. Linear regression analyses were used to assess relationships between variables.

\section{Results}

Recordings were successfully obtained from all women and all studies were included in the analysis. The mean duration of the studies was one hour approximately and they were all well tolerated (without symptoms and/or complications). The mean gestational age at examination in HP women was $34.2 \pm$ 3.3 weeks. Demographic, anthropometric, and clinical data are shown in Table 1. As was mentioned above, age, height, and cardiovascular risk factors were taken into account in order to match groups. Body mass index was significantly higher in HP compared with NP $(P<0.05)$.

Baseline cardiovascular characteristics are given and compared in Table 2. The mean heart rate was higher in pregnancy compared to the nonpregnant controls. In addition, baseline $\mathrm{pDBP}$ and MBP levels were significantly higher in NP in comparison with HP $(P<0.05)$. No pSBP differences were found between the groups. However, in addition to differences in CDBP, significantly higher values in CSBP were evidenced in NP women.

Mean AIx@75 was higher in NP with respect to HP (18.8 $\pm 10.1 \%$ versus $8.9 \pm 8.6 \%$; resp.; $P=0.019)$, whereas amplification ratio was significantly reduced (Table 2 ).

When analyzing geometrical and biomechanical characteristics of muscular peripheral arteries important differences were found between groups. In concrete, basal brachial SD and DD were significantly increased in HP versus NP $(P<$ $0.001)$ and $P W V c r$ values were higher in NP compared with HP $(P=0.003)$. Elastic arteries such as aorta and CCAs were also analyzed by both regional and local arterial stiffness parameters (Table 2). Meaningfully differences in stiffness were only evidenced in regional stiffness (PWVcf), which was significantly reduced in HP. Values of local stiffness $\left(E_{P}\right)$ of both right and left CCA did not reach statistical differences
TABLE 1: Demographic, anthropometric, and clinical characteristics.

\begin{tabular}{lccc}
\hline Variable & NP & HP & $P$ value $^{*}$ \\
\hline$N$ & 22 & 11 & \\
Age (years) & $27.9 \pm 6.2$ & $29.1 \pm 4.7$ & 0.475 \\
Gestational age (weeks) & N/A & $34.2 \pm 3.3$ & N/A \\
Weight (kg) & $61.8 \pm 7.1$ & $66.9 \pm 7.4$ & 0.065 \\
Height $(\mathrm{cm})$ & $158.6 \pm 8.2$ & $157.9 \pm 7.1$ & 0.755 \\
BMI (kg/m ${ }^{2}$ ) & $24.7 \pm 2.9$ & $27.0 \pm 3.7^{*}$ & 0.048 \\
Carotid-to-radial distance (cm) & $60.2 \pm 9.1$ & $61.6 \pm 4.2$ & 0.550 \\
Carotid-to-femoral distance (cm) & $59.0 \pm 2.6$ & $58.5 \pm 3.2$ & 0.665 \\
SSN-to-carotid distance (cm) & $8.0 \pm 1.1$ & $7.6 \pm 0.8$ & 0.262 \\
Hypertension (\%) & 0.0 & 0.0 & N/A \\
Dyslipidemia (\%) & 20.0 & 18.1 & 0.700 \\
Diabetes (\%) & 0.0 & 0.0 & N/A \\
Cardiovascular disease (\%) & 0.0 & 0.0 & N/A \\
\hline
\end{tabular}

Values are expressed as means \pm SD or as prevalence in \%. * indicates $P<$ 0.05 . All comparisons were determined using two-tailed unpaired student $t$ test and $\chi^{2}$ Test with or without Yates's correction if appropriate. $n$ : number of patients per group; N/A: not applicable; NP: nonpregnant women; HP: healthy pregnant women; BMI: body mass index; SSN: suprasternal notch.

among groups, despite the lower mean values that were found in HP.

None of the groups presented atherosclerotic plaques. Structural differences were only noticed on the right CCA, where CIMT was significantly reduced in HP with respect to NP women $(P=0.044)$.

Taking into account the VRT, all groups evoked endothelial stimulus (reactive hyperemia) evaluated by changes in SR before and after cuff deflation $(P<0.001)$ (Table 3$)$. Pregnancy was associated with increased baseline SR values in comparison with NP controls $\left(66.2 \pm 24.4 \mathrm{~s}^{-1}\right.$ versus $\left.110.8 \pm 40.1 \mathrm{~s}^{-1} ; P<0.001\right)$. Nevertheless, after cuff deflation, peak SR and $\Delta \mathrm{SR} \%$ were not different between groups $(P=0.079$ and $P=0.525$, resp. $)$, ensuring a similar hyperemic stimulus (Table 3 ). As was expected, no significant changes were found in heart rate or blood pressure intraand intergroup before and after the cuff deflation (data not shown).

Regarding the FMD, both groups showed dilatation of the brachial artery with respect to basal state, but as was expected, HP women showed quantitatively the highest FMD response $(9.6 \pm 3.4 \%$ versus $7.1 \pm 2.3 \% ; P<0.05)$ (Table 3 ), despite the higher basal brachial artery diameter existing in HP (Table 2). One minute after cuff deflation, PWVcr significantly decreased in both HP $(6.8 \pm 1.4$ to $5.8 \pm 0.9 \mathrm{~m} / \mathrm{s}$; $P=0.005)$ and NP $(8.4 \pm 1.1$ to $7.4 \pm 0.9 \mathrm{~m} / \mathrm{s} ; P<$ 0.001). The mean absolute change of the study groups was similar; however PWVcr response in \% differed comparing HP women with NP women $(-14.0 \%$ versus $-8.5 \% ; P<$ $0.05)$ (Table 3). L-FMC of the brachial artery occurred in both groups independently of the physiological status $(P<$ $0.001)$. However, even though maximal vasoconstriction of the brachial artery (negative values) was observed in HP women $(-7.0 \pm 4.7 \% ; P<0.001)$ in comparison to NP $(-5.7 \pm 2.4 \% ; P<0.001)$, the magnitude of the reduction of 
TABLE 2: Central (aortic) and peripheral arterial structural and functional parameters.

\begin{tabular}{|c|c|c|c|}
\hline Variable & NP & $\mathrm{HP}$ & $\begin{array}{c}P \\
\text { value }^{*}\end{array}$ \\
\hline Heart rate (beats/minute) & $73.8 \pm 10.8$ & $85.0 \pm 10.0^{*}$ & 0.007 \\
\hline Peripheral SBP (mmHg) & $113.0 \pm 11.2$ & $112.0 \pm 9.2$ & 0.740 \\
\hline Peripheral DBP (mmHg) & $72.4 \pm 8.2$ & $62.5 \pm 8.9^{*}$ & 0.004 \\
\hline MBP (mmHg) & $89.0 \pm 8.5$ & $78.6 \pm 5.7^{*}$ & 0.002 \\
\hline Peripheral PP (mmHg) & $45.9 \pm 9.3$ & $49.8 \pm 17.8$ & 0.235 \\
\hline Central SBP (mmHg) & $104.2 \pm 9.9$ & $95.8 \pm 6.8^{*}$ & 0.043 \\
\hline Central DBP (mmHg) & $72.2 \pm 8.2$ & $63.4 \pm 9.4^{*}$ & 0.016 \\
\hline Central PP (mmHg) & $32.0 \pm 4.3$ & $32.4 \pm 11.0$ & 0.683 \\
\hline $\begin{array}{l}\text { Amplification ratio } \\
(\mathrm{pPP} / \mathrm{cPP})\end{array}$ & $1.31 \pm 0.21$ & $1.51 \pm 0.16^{*}$ & 0.006 \\
\hline AIx@75 (\%) & $18.8 \pm 10.1$ & $8.9 \pm 8.6^{*}$ & 0.019 \\
\hline $\begin{array}{l}\text { Carotid-to-radial PWV } \\
(\mathrm{m} / \mathrm{s})\end{array}$ & $8.4 \pm 1.1$ & $6.8 \pm 1.4^{*}$ & 0.003 \\
\hline Brachial SD (mm) & $3.1 \pm 0.3$ & $3.9 \pm 0.3^{*}$ & $<0.001$ \\
\hline Brachial DD (mm) & $2.9 \pm 0.3$ & $3.7 \pm 0.3^{*}$ & $<0.001$ \\
\hline $\begin{array}{l}\text { Carotid-to-femoral PWV } \\
(\mathrm{m} / \mathrm{s})\end{array}$ & & & 0.022 \\
\hline Right CCA SD (mm) & $6.8 \pm 0.6$ & & 0.311 \\
\hline Right CCA DD (mm) & $6.7 \pm 0.5$ & $6.9 \pm 0.7$ & 0.388 \\
\hline Right CCA $E_{P}(\mathrm{mmHg})$ & $416.9 \pm 157.6$ & $350.6 \pm 110.0$ & 0.301 \\
\hline Right CIMT (mm) & $0.550 \pm 0.077$ & $0.462 \pm 0.089^{*}$ & 0.044 \\
\hline Left CCA SD (mm) & $6.6 \pm 0.6$ & $7.2 \pm 0.4^{*}$ & 0.040 \\
\hline Left CCA DD (mm) & $6.0 \pm 0.5$ & $6.5 \pm 0.3$ & 0.062 \\
\hline Left CCA $E_{P}(\mathrm{mmHg})$ & $401.8 \pm 155.7$ & $352.7 \pm 179.0$ & 0.702 \\
\hline Left CIMT (mm) & $0.572 \pm 0.090$ & $0.519 \pm 0.080$ & 0.146 \\
\hline
\end{tabular}

Values are expressed as means \pm SD. $*$ indicates $P<0.05$. All comparisons were determined using two-tailed unpaired Student $t$-test. NP: nonpregnant women; HP: healthy pregnant women; SBP, DBP, MBP, and PP: systolic, diastolic, mean, and pulse pressure, respectively. pPP and cPP: peripheral and central pulse pressure, respectively. AIx@75: augmentation index adjusted to a heart rate of 75 beats/minute; PWV: pulse wave velocity; SD and DD: systolic and diastolic diameter, respectively; $E_{P}$ : Peterson's or pressure-strain elastic modulus; CCA: common carotid artery; CIMT: carotid intima-media thickness.

TABLE 3: Vascular reactivity parameters: endothelial function.

\begin{tabular}{lccc}
\hline Variable & NP & HP & $P$ value $^{*}$ \\
\hline Basal SR $\left(\mathrm{s}^{-1}\right)$ & $66.2 \pm 24.4$ & $110.8 \pm 40.1^{*}$ & $<0.001$ \\
Peak SR $\left(\mathrm{s}^{-1}\right)$ & $180.0 \pm 73.7$ & $227.0 \pm 67.8$ & 0.079 \\
$\Delta$ SR $(\%)$ & $132.5 \pm 59.9$ & $110.9 \pm 88.5$ & 0.525 \\
\hline FMD (\%) & $7.1 \pm 2.3$ & $9.6 \pm 3.4^{*}$ & 0.039 \\
$\Delta$ PWVcr (\%) & $-8.5 \pm 6.4$ & $-14.0 \pm 7.8^{*}$ & 0.035 \\
L-FMC $(\%)$ & $-5.7 \pm 2.4$ & $-7.0 \pm 4.7$ & 0.208 \\
\hline
\end{tabular}

Values are expressed as means \pm SD. $*$ indicates $P<0.05$. All comparisons were determined using two-tailed unpaired Student $t$-test. NP: nonpregnant women; HP: healthy pregnant women; FMD: flow-mediated dilation; LFMC: low-flow-mediated constriction; PWVcr: carotid-to-radial pulse wave velocity; SR: shear rate. arterial diameters was not statistically different between the groups (Table 3 ).

Finally, there were no significant correlations between endothelial function parameters (FMD, L-FMC, or $\triangle \mathrm{PWVcr} \%$ ) and AIx@75, PWVcf, or central blood pressure (data not shown). However, FMD correlated with L-FMC $(R=0.54, P=0.038)$ and $\Delta \mathrm{PWVcr} \%(R=0.419, P=0.037)$, whereas no significant correlation was evidenced between $\triangle \mathrm{PWV}$ cr\% and L-FMC $(R=0.30, P=0.198)$. A positive correlation between L-FMC (negative number) and basal SR (positive number) was found across the whole studied population $(R=0.587, P=0.017)$.

\section{Discussion}

The present study is, to our knowledge, the first one to determine and assess simultaneously, in a group of healthy pregnant women, the endothelial function by using three different but complementary methods in conjunction with the determination of central and peripheral structural and functional arterial validated parameters. These approaches allow us to conclude that, with respect to nonpregnant women matched by age, anthropometric features and cardiovascular risk factors, pregnant women showed (1) reduced aortic and "upper limb" arterial stiffness levels, in coherence with the higher basal brachial artery diameters that were found in this group; (2) reduced central (aortic), but not peripheral, systolic blood pressure, determined by a reduced contribution of reflected waves to central aortic pressure waveform (lower AIx@75); (3) an enhanced recruitable (FMD), but not resting (L-FMC), endothelial function, despite higher basal brachial diameters.

4.1. Physiological Considerations. Regarding the hemodynamic parameters, we found that HP women showed, in comparison to controls, increased HR and reduced pDBP and $\mathrm{MBP}$ in basal state. These findings are in consonance with an expected pregnancy-induced decrease in peripheral vascular resistance and increased cardiac output at rest. However, pregnancy-related changes were also notable when central hemodynamics is analyzed. Central (aortic) systolic blood pressure and PWVcf (aortic stiffness) were lower in HP comparing to controls, independently of brachial systolic blood pressure levels. In addition, AIx@75, a composite parameter of systemic arterial stiffness and wave reflection amplitude, was different between HP and NP. This suggests that healthy pregnancy is associated with reduced wave reflection contribution to the central aortic pressure waveform and central arterial (aortic) stiffness. However, changes in CCA stiffness $\left(E_{p}\right)$ related with pregnancy were not statistically significant, despite lower mean levels in HP. This finding supports Kärkkäinen et al. report, since these authors evidenced that carotid arterial distensibility (inverse of $E_{P}$ ) decreased towards the end of the pregnancy reaching the lowest values in the third trimester [27]. Nevertheless, taking our results together, we evidenced that healthy pregnancy is associated with reduced aortic stiffness, central systolic pressure, and wave reflections. 
Among the methods that allow for measurement of endothelial function in the clinical setting, FMD has rapidly gained popularity because of its simplicity, reproducibility, and noninvasiveness [2, 28]. However, as was mentioned earlier, one important limitation of FMD is that it only provides information about the "recruitability" of endothelial function (i.e., its responsiveness to a specific stimulus) and not about "resting" endothelial function (i.e., release of endothelial autacoids before FMD measures are initiated) [8]. We here analyze, in healthy pregnant women, both types of functional aspects of endothelial function, "endothelial recruitability" through FMD and PWVcr changes and "resting endothelial tone" through L-FMC. As it was expected, the magnitude of FMD observed in HP in response to VRT surpassed that observed in NP. This finding, which is similar to that described in previous reports, is in coherence with an enhanced endothelial function assessed by this method $[6,7]$. On another side, when analyzing changes in arterial stiffness due to VRT, even though both groups showed a reduction in PWVcr values, HP showed the major decrease $(P<0.05)$. It is noteworthy that both groups showed a similar "endothelial stimulus," since peak SR and $\Delta$ SR\% were similar between them. In addition, even though starting (basal) levels of brachial diameter and PWVcr (basal state) in HP were higher and lower, respectively, these values were not correlated with the vascular response (i.e., FMD and $\triangle \mathrm{PWVcr} \%$ ). When examining the relationship between FMD and PWVcr the analysis should take into account the Moens and Korteweg equation. In that sense, PWV is determined by both arterial diameter and the elastic modulus, among other factors [12]. In a previous study, we evidenced in healthy subjects that changes in PWVcr due to the VRT may be also provoked by a smooth muscle relaxation. If there were a right shift in the brachial pressure-volume loop, post-cuff deflation, in addition to a slope decrease due to smooth muscle relaxation, the global response might suit our results (as PWVcr changes were even higher than those only expected by changes in VMF) [3]. In light of our results, we hypothesize that besides an enhanced vasodilatory response, smooth muscle relaxation could be pronounced in healthy pregnancy.

Taking into account "resting" endothelial tone, our results show that, during the cuff inflation, L-FMC occurred in the brachial artery independently of the physiological status. However, mean L-FMC was not different between the groups. Although L-FMC was firstly described at the radial artery as a specific phenomenon [9], in the 90's it had been already reported in the brachial artery in response to the cuff occlusion in subjects with hypercholesterolemia [33]. Later, Spiro et al. evidenced that this specific phenomenon also occurs in healthy subjects at the brachial artery and it can be measured reliably [34]. Studies agree that vasoconstriction of the radial artery occurs during the cuff inflation in nonpregnant women, although the mechanisms involved remain not completely understood $[9,35,36]$. L-FMC of the brachial artery has been controversial and several studies demonstrated conflicting results $[33-35,37,38]$. Indeed, Weissgerber et al. did not evidence L-FMC in the brachial artery in pregnant women [35], in contraposition with our results. Differences in cardiovascular profile, technical aspects, methodological issues, and/or interobserver variability could explain the widely variable results, as it occurs with FMD measurements [39-41]. For example, we here measure L-FMC of the brachial artery in a regimen of low but not zero blood flow (as it occurs in the radial artery) in a level that is upstream of the occlusion site. Therefore, the magnitude of reduced blood flow in the brachial artery and its relationship with the basal levels (endothelial "negative" stimulus for vasoconstriction) should surely yield different brachial responses. Nevertheless, our results indicate that the more the basal blood flow (or $\mathrm{SR}$ ), the more the vasoconstriction of the brachial artery provoked by the occlusion of the pneumatic cuff. Even though we did not measure "the residual" brachial blood flow during the cuff occlusion, it is expected that the absolute change in brachial blood flow may be of greater amount with the same occlusion protocol, if the starting point of blood flow in basal conditions is increased (i.e., increased "negative" stimulus for vasoconstriction).

We found a significant correlation between FMD and L-FMC, FMD and $\triangle \mathrm{PWVcr} \%$, but not between L-FMC and $\triangle \mathrm{PWVcr} \%$. Our results indicate that brachial artery responses to inflation and deflation of the cuff related with endothelial dynamics could share some vascular mechanism. However, there are confusing results around the FMD and L-FMC correlation, with variable results depending on the analyzed artery (brachial versus radial) and type of physiological or pathophysiological circumstance [8-10, 34]. This emphasizes again the complexity of studying "endothelial functions." Although both L-FMC and FMD are an expression of the vascular reactivity in response to changes in blood flow, their relationship is neither conceptually simple nor mathematically linear [10]. Nevertheless, it is reasonable to think that the same vasodilatatory mechanisms (i.e., nitric oxide) involved in response to increased blood flow (shear stress) will diminish (with the consequent vasoconstriction) when the stimulus for its production is reduced/abolish.

4.2. Interpretation of Findings. The important additional information brought by the introduction of changes in PWVcr and L-FMC, together with the information of central and peripheral hemodynamics, is that these variables provide information concerning different aspects of vascular reactivity and endothelial function, therefore complementing (and not overlapping) the information provided by FMD. An enhanced response and/or increased vasodilator reserve to changes in blood flow in a concrete vascular ledge (i.e., brachial artery) implicates an elevated capability of the arterial system to accomplish an appropriate vascular adjustment against hemodynamic changes in the long term (fetal growth) and even in the short term (exercise, change of position, etc.). In addition, these could be associated with cardiovascular benefits reported by other authors like reduced left ventricle afterload and improve diastolic function and reduced myocardial oxygen demand in the maternal circulation [42-44]. Previous results of our group suggested that pregnancy-induced hypertension (i.e., preeclampsiaeclampsia syndrome) could be associated with increased 
central aortic pressure, elastic arteries stiffness, and wave reflections, in conjunction with both resting and recruitable endothelial dysfunctions [45]. These arterial disturbances would not only blunt the mentioned hemodynamic benefits of the pregnancy physiological condition but also add extra load to the maternal circulation in the context of increased cardiac output and fetal requirements. However, although this comprehensive arterial assessment would improve our understanding of the haemodynamics of both healthy pregnancy and pregnancy-related diseases, the inclusion of this information together with the recognized validated clinical, obstetric, and laboratory variables remains to be addressed during the first trimester of pregnancy, since at this time they could have an additional/complementary value in the prediction of preeclampsia $[46,47]$. In this small study, that addresses the feasibility of measuring these parameters simultaneously, simply, and noninvasively, we found encouraging results that, we believe, warrant further investigation.

4.3. Limitations and Perspectives. The sample size of our study was relatively small. However, our findings were statistically significant and, by definition, this indicates that the study was adequately statistically powered. Our technical approaches including the use of both multiple types of automated and semiautomated edge-detection/point software in ultrasound image and pressure wave assessment are largely operator independent and also empower our findings [28]. Given the means of the different variables and SDs observed in previous works and in the present sample, twenty-eight subjects $(n=$ 28 ) of the total sample size (the sum of the sizes of comparison groups) would be required to detect a statistically significant effect of the pregnancy status with at least $80 \%$ of power [45]. Secondly, even groups were properly matched, women with dyslipidemia were included in the analysis, and this could have an impact in our results, being a limitation of the "healthy groups."

This vascular approach may provide a more comprehensive assessment of vascular state and endothelial function in normal pregnancy. Future studies will have to determine if accounting of this information, particularly during the early stages of pregnancy (i.e., first trimester), in conjunction with recognized important clinical, obstetric, laboratory variables, will be able to improve early detection of pathophysiological circumstances like pregnancy-induced hypertension.

\section{Conclusion}

With respect to nonpregnant women matched by age, anthropometric features and cardiovascular risk factors, pregnant women showed (1) reduced aortic and "upper limb" arterial stiffness levels, in coherence with the higher basal brachial artery diameters that were found in this group; (2) reduced central (aortic), but not peripheral, systolic blood pressure, determined by a reduced contribution of reflected waves to central aortic pressure waveform; and (3) an enhanced recruitable, but not resting, endothelial function, despite higher basal brachial diameters.

\section{Conflict of Interests}

The authors declare that there is no conflict of interests regarding the publication of this paper.

\section{Acknowledgments}

The authors wish to acknowledge the technical assistance given by Manuela Pereira. This work was supported by the CUiiDARTE Centre and Project, Agencia Nacional de Investigación e Innovación (ANII-Uruguay), Comisión Sectorial de Investigación Científica and Espacio Interdisciplinario (Universidad de la República).

\section{References}

[1] D. S. Celermajer, K. E. Sorensen, V. M. Gooch et al., "Noninvasive detection of endothelial dysfunction in children and adults at risk of atherosclerosis," The Lancet, vol. 340, no. 8828, pp. 1111-1115, 1992.

[2] M. C. Corretti, T. J. Anderson, E. J. Benjamin et al., "Guidelines for the ultrasound assessment of endothelial-dependent flowmediated vasodilation of the brachial artery: a report of the International Brachial Artery Reactivity Task Force," Journal of the American College of Cardiology, vol. 39, no. 2, pp. 257-265, 2002, Erratum in: Journal of the American College of Cardiology, vol. 39, no. 6, p. 1082, 2002.

[3] J. Torrado, D. Bia, Y. Zocalo et al., "Reactive hyperemia-related changes in carotid-radial pulse wave velocity as a potential tool to characterize the endothelial dynamics," in Proceedings of the Annual International Conference of the IEEE Engineering in Medicine and Biology Society (EMBC '09), pp. 1800-1803, IEEE, Minneapolis, Minn, USA, September 2009.

[4] B. Takase, T. Goto, A. Hamabe et al., "Flow-mediated dilation in brachial artery in the second half of pregnancy and prediction of pre-eclampsia," Journal of Human Hypertension, vol. 17, no. 10, pp. 697-704, 2003.

[5] E. V. D. C. Filho, C. Mohr, B. J. A. Filho et al., "Flowmediated dilatation in the differential diagnosis of preeclampsia syndrome," Arquivos Brasileiros de Cardiologia, vol. 94, no. 2, pp. 182-186, 2010.

[6] I. Dørup, K. Skajaa, and K. E. Sørensen, "Normal pregnancy is associated with enhanced endothelium-dependent flowmediated vasodilation," The American Journal of PhysiologyHeart and Circulatory Physiology, vol. 276, no. 3, part 2, pp. H821-H825, 1999.

[7] M. D. Savvidou, N. A. Kametas, A. E. Donald, and K. H. Nicolaides, "Non-invasive assessment of endothelial function in normal pregnancy," Ultrasound in Obstetrics and Gynecology, vol. 15, no. 6, pp. 502-507, 2000.

[8] T. Gori, J. D. Parker, and T. Münzel, "Flow-mediated constriction: further insight into a new measure of vascular function," European Heart Journal, vol. 32, no. 7, pp. 784-787, 2011.

[9] T. Gori, S. Dragoni, M. Lisi et al., "Conduit artery constriction mediated by low flow. A novel noninvasive method for the assessment of vascular function," Journal of the American College of Cardiology, vol. 51, no. 20, pp. 1953-1958, 2008.

[10] T. Gori, S. Grotti, S. Dragoni et al., "Assessment of vascular function: flow-mediated constriction complements the information of flow-mediated dilatation," Heart, vol. 96, no. 2, pp. 141-147, 2010. 
[11] K. K. Naka, A. C. Tweddel, S. N. Doshi, J. Goodfellow, and A. H. Henderson, "Flow-mediated changes in pulse wave velocity: a new clinical measure of endothelial function," European Heart Journal, vol. 27, no. 3, pp. 302-309, 2006.

[12] S. Laurent, J. Cockcroft, L. Van Bortel et al., "Expert consensus document on arterial stiffness: methodological issues and clinical applications," European Heart Journal, vol. 27, no. 21, pp. 2588-2605, 2006.

[13] L. M. Van Bortel, S. Laurent, P. Boutouyrie et al., "Expert consensus document on the measurement of aortic stiffness in daily practice using carotid-femoral pulse wave velocity," Journal of Hypertension, vol. 30, no. 3, pp. 445-448, 2012.

[14] H. Kamran, L. Salciccioli, Eun Hee Ko et al., "Effect of reactive hyperemia on carotid-radial pulse wave velocity in hypertensive participants and direct comparison with flow-mediated dilation: a pilot study," Angiology, vol. 61, no. 1, pp. 100-106, 2010.

[15] J. Torrado, I. Farro, F. Farro et al., "Carotid-radial pulse wave velocity as an alternative tool for the evaluation of endothelial function during pregnancy: potential role in identifying hypertensive disorders of pregnancy," in Proceedings of the Annual International Conference of the IEEE Engineering in Medicine and Biology Society (EMBC '12), pp. 5603-5606, IEEE, San Diego, Calif, USA, August-September 2012.

[16] J. P. van den Wijngaard, B. E. Westerhof, D. J. Faber, M. M. Ramsay, N. Westerhof, and M. J. van Gemert, "Abnormal arterial flows by a distributed model of the fetal circulation," American Journal of Physiology-Regulatory, Integrative and Comparative Physiology, vol. 291, no. 5, pp. R1222-R1233, 2006, Erratum in: American Journal of Physiology-Regulatory, Integrative and Comparative Physiology, vol. 292, no. 1, p. R663, 2007.

[17] J. S. Morton and S. T. Davidge, "Arterial endothelium-derived hyperpolarization: potential role in pregnancy adaptations and complications," Journal of Cardiovascular Pharmacology, vol. 61, no. 3, pp. 197-203, 2013.

[18] D. B. Santana, Y. A. Zócalo, and R. L. Armentano, "Integrated e-health approach based on vascular ultrasound and pulse wave analysis for asymptomatic atherosclerosis detection and cardiovascular risk stratification in the community," IEEE Transactions on Information Technology in Biomedicine, vol. 16, no. 2, pp. 287-294, 2012.

[19] D. Bia, Y. Zócalo, I. Farro et al., "Integrated evaluation of agerelated changes in structural and functional vascular parameters used to assess arterial aging, subclinical atherosclerosis, and cardiovascular risk in uruguayan adults: CUiiDARTE project," International Journal of Hypertension, vol. 2011, Article ID 587303, 112 pages, 2011.

[20] D. B. Santana, Y. A. Zócalo, I. F. Ventura et al., "Health informatics design for assisted diagnosis of subclinical atherosclerosis, structural, and functional arterial age calculus and patientspecific cardiovascular risk evaluation," IEEE Transactions on Information Technology in Biomedicine, vol. 16, no. 5, pp. 943951, 2012.

[21] National Collaborating Centre for Women's and Children's Health, Hypertension in Pregnancy. The Management of Hypertensive Disorders During Pregnancy, Clinical Guideline; no. 107, National Institute for Health and Clinical Excellence (NICE), London, UK, 2010.

[22] National Collaborating Centre for Women's and Children's Health, Diabetes in Pregnancy: Management of Diabetes and Its Complications from Preconception to the Postnatal Period, (Clinical Guideline), National Collaborating CentreNational Institute for Health and Clinical Excellence (NICE), London, UK, 2015.
[23] Y. C. Chiu, P. W. Arand, S. G. Shroff, T. Feldman, and J. D. Carroll, "Determination of pulse wave velocities with computerized algorithms," American Heart Journal, vol. 121, no. 5, pp. 14601470, 1991.

[24] A. L. Pauca, M. F. O’Rourke, and N. D. Kon, "Prospective evaluation of a method for estimating ascending aortic pressure from the radial artery pressure waveform," Hypertension, vol. 38, no. 4, pp. 932-937, 2001.

[25] J. H. Stein, C. E. Korcarz, R. T. Hurst et al., "Use of carotid ultrasound to identify subclinical vascular disease and evaluate cardiovascular disease risk: a consensus statement from the American society of echocardiography carotid intima-media thickness task force endorsed by the society for vascular medicine," Journal of the American Society of Echocardiography, vol. 21, no. 2, pp. 93-111, 2008.

[26] M. F. O’Rourke, J. A. Staessen, C. Vlachopoulos, D. Duprez, and G. E. Plante, "Clinical applications of arterial stiffness; definitions and reference values," American Journal of Hypertension, vol. 15, no. 5, pp. 426-444, 2002.

[27] H. Kärkkäinen, H. Saarelainen, P. Valtonen et al., "Carotid artery elasticity decreases during pregnancy-the Cardiovascular Risk in Young Finns study," BMC Pregnancy and Childbirth, vol. 14, article 98, 2014.

[28] D. H. J. Thijssen, M. A. Black, K. E. Pyke et al., "Assessment of flow-mediated dilation in humans: a methodological and physiological guideline," The American Journal of PhysiologyHeart and Circulatory Physiology, vol. 300, no. 1, pp. H2-H12, 2011.

[29] D. Craiem, G. Chironi, A. Simon, and J. Levenson, "New assessment of endothelium-dependent flow-mediated vasodilation to characterize endothelium dysfunction," American Journal of Therapeutics, vol. 15, no. 4, pp. 340-344, 2008.

[30] K. E. Pyke, E. M. Dwyer, and M. E. Tschakovsky, "Impact of controlling shear rate on flow-mediated dilation responses in the brachial artery of humans," Journal of Applied Physiology, vol. 97, no. 2, pp. 499-508, 2004.

[31] J. Torrado, D. Bia, Y. Zócalo et al., "Carotid-radial pulse wave velocity as a discriminator of intrinsic wall alterations during evaluation of endothelial function by flow-mediated dilatation," in Proceedings of the Annual International Conference of the IEEE Engineering in Medicine and Biology Society (EMBC '11), pp. 6458-6461, IEEE, Boston, Mass, USA, August-September 2011.

[32] J. Torrado, D. Bia, Y. Zócalo, I. Farro, F. Farro, and R. L. Armentano, "Hyperemia-related changes in arterial stiffness: comparison between pulse wave velocity and stiffness index in the vascular reactivity assessment," International Journal of Vascular Medicine, vol. 2012, Article ID 490742, 7 pages, 2012.

[33] V. Filitti, P. Giral, A. Simon, I. Merli, M. Del Pino, and J. Levenson, "Enhanced constriction of the peripheral large artery in response to acute induction of a low-flow state in human hypercholesterolemia," Arteriosclerosis and Thrombosis, vol. 11, no. 1, pp. 161-166, 1991.

[34] J. R. Spiro, J. E. Digby, G. Ghimire et al., "Brachial artery lowflow-mediated constriction is increased early after coronary intervention and reduces during recovery after acute coronary syndrome: characterization of a recently described index of vascular function," European Heart Journal, vol. 32, no. 7, pp. 856-866, 2011.

[35] T. L. Weissgerber, G. A. L. Davies, and M. E. Tschakovsky, "Low flow-mediated constriction occurs in the radial but not the 
brachial artery in healthy pregnant and nonpregnant women," Journal of Applied Physiology, vol. 108, no. 5, pp. 1097-1105, 2010.

[36] E. A. Dawson, A. Alkarmi, D. H. J. Thijssen et al., "Low-flow mediated constriction is endothelium-dependent: effects of exercise training after radial artery catheterization," Circulation: Cardiovascular Interventions, vol. 5, no. 5, pp. 713-719, 2012.

[37] B. A. Parker, S. J. Ridout, and D. N. Proctor, "Age and flowmediated dilation: a comparison of dilatory responsiveness in the brachial and popliteal arteries," American Journal of Physiology-Heart and Circulatory Physiology, vol. 291, no. 6, pp. H3043-H3049, 2006.

[38] K. E. Pyke and M. E. Tschakovsky, "Peak vs. total reactive hyperemia: which determines the magnitude of flow-mediated dilation?" Journal of Applied Physiology, vol. 102, no. 4, pp. 15101519, 2007.

[39] D. H. J. Thijssen, M. M. van Bemmel, L. M. Bullens et al., “The impact of baseline diameter on flow-mediated dilation differs in young and older humans," The American Journal of PhysiologyHeart and Circulatory Physiology, vol. 295, no. 4, pp. H1594H1598, 2008.

[40] M. L. Bots, J. Westerink, T. J. Rabelink, and E. J. P. De Koning, "Assessment of flow-mediated vasodilatation (FMD) of the brachial artery: effects of technical aspects of the FMD measurement on the FMD response," European Heart Journal, vol. 26, no. 4, pp. 363-368, 2005.

[41] K. E. Pyke and M. E. Tschakovsky, "The relationship between shear stress and flow-mediated dilatation: implications for the assessment of endothelial function," The Journal of Physiology, vol. 568, no. 2, pp. 357-369, 2005.

[42] S. H. Kubo, T. S. Rector, A. J. Bank, R. E. Williams, and S. M. Heifetz, "Endothelium-dependent vasodilation is attenuated in patients with heart failure," Circulation, vol. 84, no. 4, pp. 1589$1596,1991$.

[43] M. Spasojevic, S. A. Smith, J. M. Morris, and E. D. M. Gallery, "Peripheral arterial pulse wave analysis in women with preeclampsia and gestational hypertension," BJOG, vol. 112, no. 11, pp. 1475-1478, 2005.

[44] E. V. Tyldum, B. Backe, A. Støylen, and S. A. Slørdahl, "Maternal left ventricular and endothelial functions in preeclampsia," Acta Obstetricia et Gynecologica Scandinavica, vol. 91, no. 5, pp. 566$573,2012$.

[45] J. Torrado, I. Farro, Y. Zócalo et al., "Preeclampsia is associated with increased central aortic pressure, elastic arteries stiffness and wave reflections, and resting and recruitable endothelial dysfunction," International Journal of Hypertension, vol. 2015, Article ID 720683, 12 pages, 2015.

[46] K. H. Nicolaides, R. Bindra, O. M. Turan et al., "A novel approach to first-trimester screening for early pre-eclampsia combining serum PP-13 and Doppler ultrasound," Ultrasound in Obstetrics and Gynecology, vol. 27, no. 1, pp. 13-17, 2006.

[47] E. Scazzocchio and F. Figueras, "Contemporary prediction of preeclampsia," Current Opinion in Obstetrics \& Gynecology, vol. 23, no. 2, pp. 65-71, 2011. 


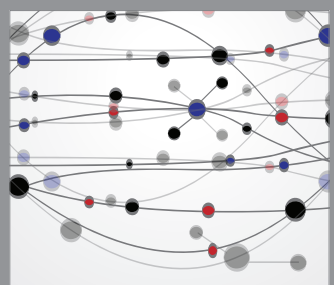

The Scientific World Journal
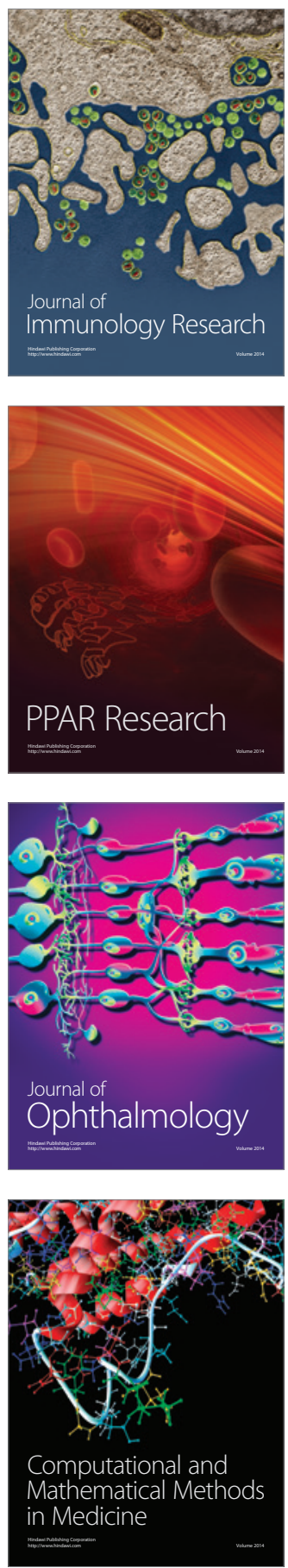

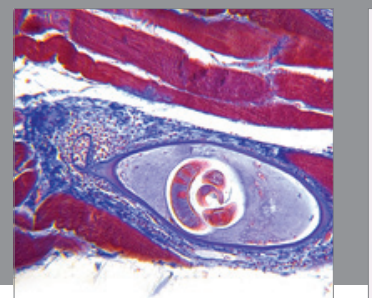

Gastroenterology

Research and Practice
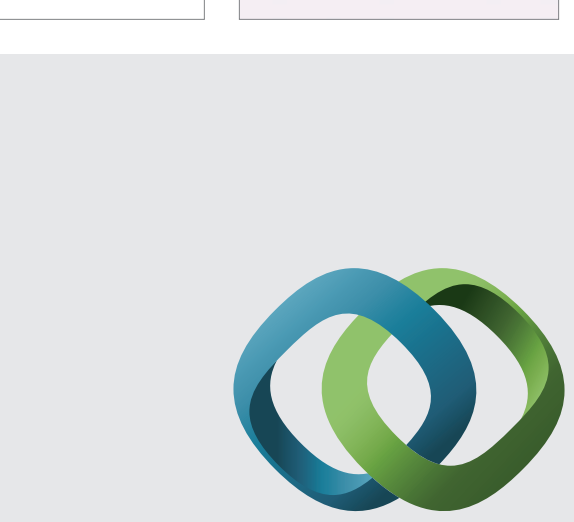

\section{Hindawi}

Submit your manuscripts at

http://www.hindawi.com
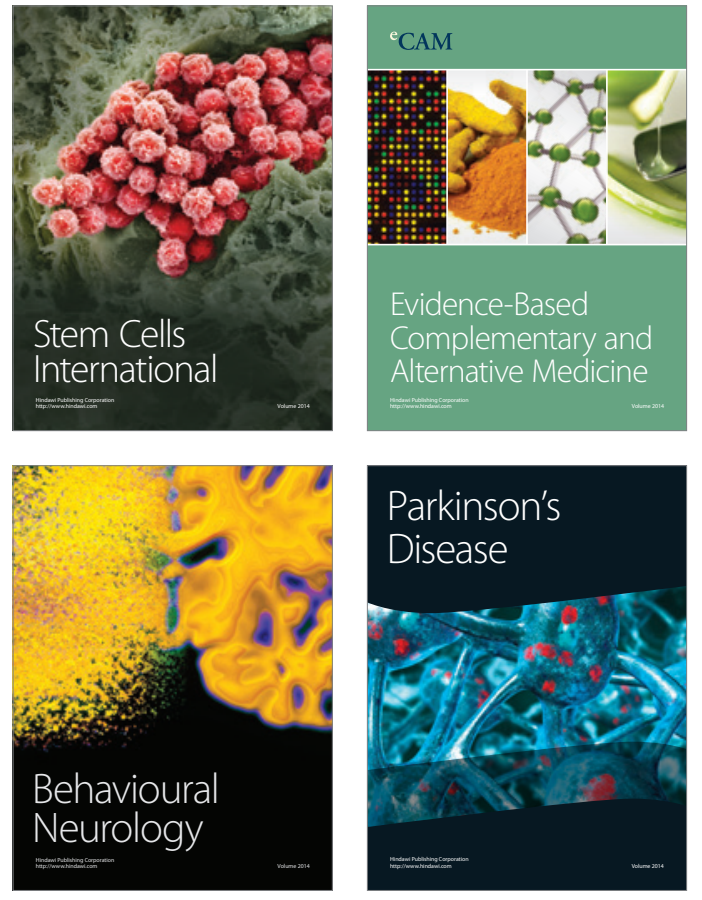
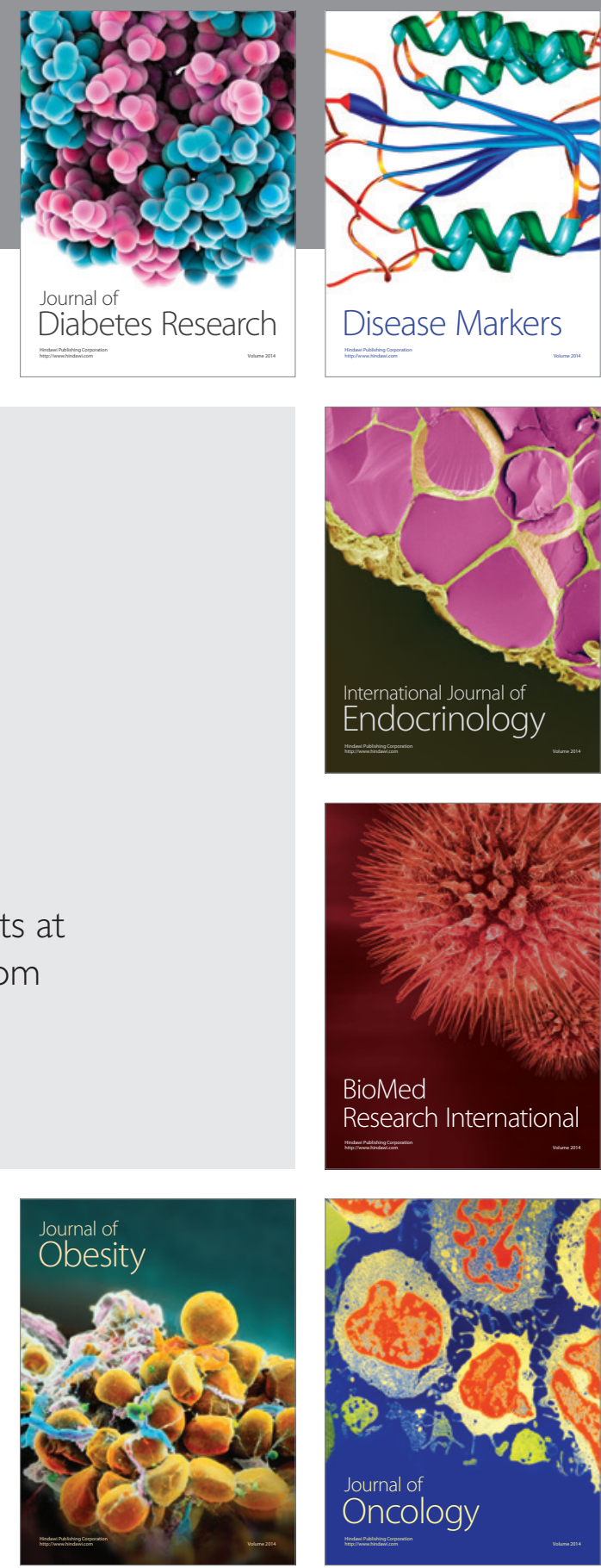

Disease Markers
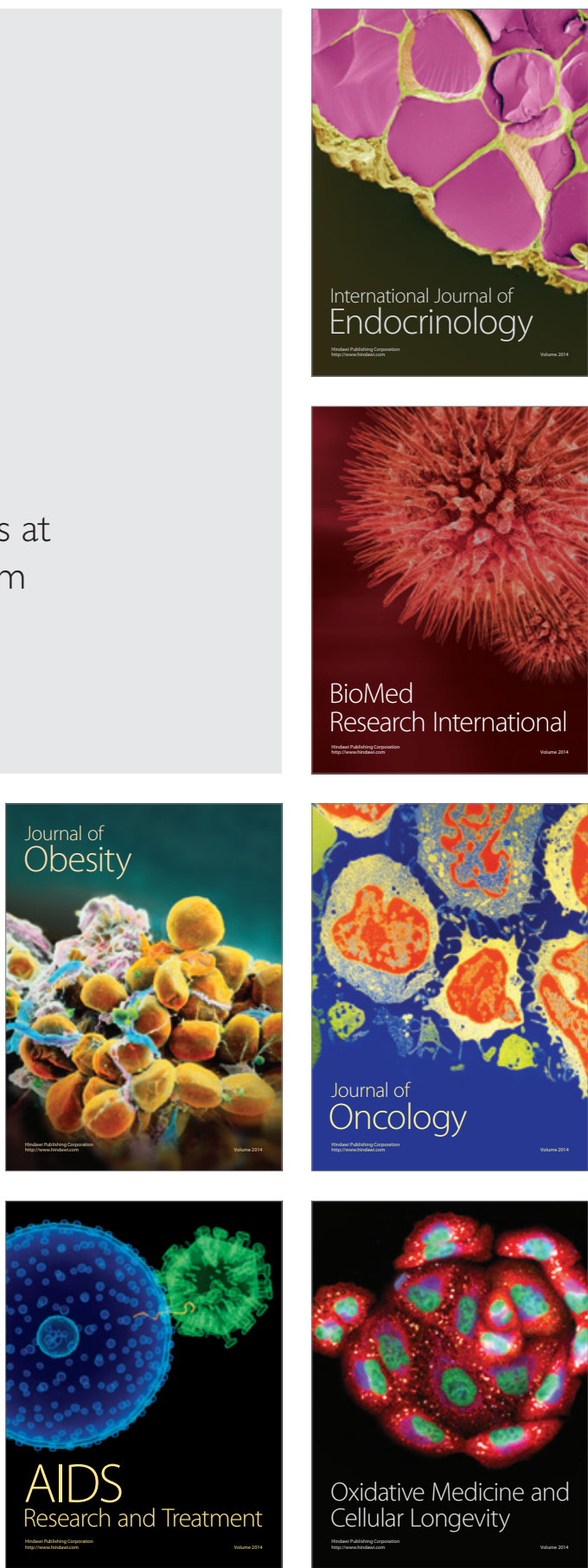